\title{
SETS OF $k$-EXTENT IN $n$-DIMENSIONAL SPACE $\dagger$
}

BY

\author{
R. L. JEFFERY
}

1. Introduction. Let $A$ be any point set on the bounded $n$-dimensional domain $D$. In his development of the theory of measure C. Carathéodory $\ddagger$ has defined in connection with the set $A$ a measurable set $\bar{A}$ which has come to be called the massgleiche Hïlle of $A . \S$

Let $B_{n}$ be a sequence of open sets containing $A$ such that $B_{n} \supset B_{n+1}$, and $\lim \mu B_{n}=\mu^{*} A$. Then the set

$$
\bar{A}=B_{1} B_{2} \ldots
$$

contains $A$, and is measurable with

$$
\mu \bar{A}=\mu^{*} A .
$$

The number of ways of selecting each $B_{n}$ is more than countable, and no rule is given for any particular choice. Consequently it is impossible to say of every point of the domain $D$ whether or not it belongs to the set $\bar{A}$. This amounts to saying that the set $\bar{A}$ is not well-defined.

It is possible to replace the set $A$ by a set $A^{\prime}$ which is effectively defined. Let $B$ be the complement of $A$ on $D$. Let $\omega_{k}$ be a sequence of cells with a point $b$ of $B$ as center, and with equal side lengths tending to zero as $k$ increases. Let

$$
\rho\left(b, \omega_{k}\right)=\frac{\mu^{*} A \omega_{k}}{\mu^{*} B \omega_{k}} .
$$

Since at each point of $B$ except at most a null set the outer metric density of $B$ is unity, it follows that $\rho\left(b, \omega_{k}\right)$ is defined for all values of $k$ at almost all points of $B$. Let $C$ be the part of $B$ for which $\rho\left(b, \omega_{k}\right)$ is defined for all values of $k$, and for which

The set

$$
\varlimsup_{k \rightarrow \infty} \rho\left(b, \omega_{k}\right)>0 .
$$

$$
A^{\prime}=A+C
$$

$\dagger$ Presented to the Society, March 25, 1932; received by the editors June 14, 1932, and, in revised form, November 18, 1932.

$\ddagger$ Über das lineare Mass von Punktmengen, Göttinger Nachrichten, 1914.

$\S$ Hahn, Theorie der Reellen Funktionen, p. 435. Caratheodory, Vorlesungen über reelle Funktionen, p. 260. 
contains $A$, and is effectively defined in terms of $A$. We also have the following:

I. The set $A^{\prime}$ is measurable in the sense of Carathéodory, and

$$
\mu A^{\prime}=\mu^{*} A .
$$

II. A necessary and sufficient condition that $A$ be measurable is that

$$
\mu^{*} C=0 .
$$

Though not explicitly stated, the proofs of I and II are contained in a previous discussion. $\dagger$

An analogous situation exists in connection with plane sets of linear extent. Let $A$ be such a set with finite outer linear measureł equal to $l$. Let $U_{n}=u_{n 1}, u_{n 2}, \cdots$ be a sequence of open convex areas which contains $A$, with $d_{n i}$, the greatest diameter of $u_{n i}$, tending to zero, and with $\sum d_{n i}$ tending to $l$. Then the set

$$
\bar{A}=U_{1} U_{2} \ldots
$$

contains $A$, is linearly measurable, $\S$ and

$$
L \bar{A}=L^{*} A .
$$

In this case too there is no way to determine for each point of the domain $D$ containing $A$ whether or not it belongs to the set $\bar{A}$. In the present paper we determine a set $A^{\prime}$ which contains the plane set of linear extent $A$, which is linearly measurable, with

$$
L A^{\prime}=L^{*} A,
$$

and which is well-defined in terms of $A$.

That these sets $A^{\prime}$ are well-defined has some significance. $\|$ A more important consideration is, however, that the concepts involved in and leading up to their definition combine to form an elegant and very useful tool for handling certain types of problems. T We do not restrict ourselves to plane sets, but carry through the discussion for sets of extent $k$ in $n$-dimensional space. We show that such sets have properties of density similar to the properties of density which Besicovitch $\dagger \dagger$ and Sierpinskiłt respectively have shown to hold

† Annals of Mathematics, (2), vol. 33, pp. 449-451.

‡ Carathéodory, Göttinger Nachrichten, loc. cit., \$23.

\& Carathéodory, Göttinger Nachrichten, loc. cit., \$28.

II Sierpinski, Fundamenta Mathematicae, vol. 2, pp. 112.

If See Annals of Mathematics, (2), vol. 33, pp. 452-459, these Transactions, vol. 34, p. 650, also the concluding section of this paper.

†† Mathematische Annalen, vol. 98, p. 422.

$\ddagger \ddagger$ Fundamenta Mathematicae, vol. 9, p. 172. 
for linearly measurable plane sets, and for plane sets which are not necessarily linearly measurable but which have linear extent. Although there would be no difficulty in giving independent proofs of the various results, to conserve space we have, whenever possible, based our proofs on those of Carathéodory and Besicovitch.

Let $S_{n}$ be the $n$-dimensional euclidean space, and $S_{k}$ a $k$-dimensional flat space $\dagger$ in $S_{n}$. Let $U$ be an open convexं domain of $S_{n}$. For a given $U$ let $S_{k}$ be such that the $k$-dimensional measure of $S_{k} U$ is a maximum, and denote this maximum measure by $l_{k}$. We shall call $l_{1}$ the greatest diameter of $U$, and denote it by $d$. Let $A$ be any bounded set in $S_{n}$, and $\rho$ any positive number. Put $A$ in a countable set of open convex domains $u_{i}$. Let $L_{k^{p}}(A)$ be the lower bound of $\sum l_{k}^{i}$ for all possible such enclosures with $d_{i}<\rho$. Evidently $L_{k}{ }^{\rho}(A)$ does not decrease as $\rho$ decreases. Let

$$
L_{k}(A)=\lim _{\rho \rightarrow 0} L_{k}^{\rho}(A) .
$$

It is clear that $L_{k}(A) \geqq 0$, and may be-infinite.

The largest value of $k$ for which $L_{k}(A) \neq 0$ determines the extent of the set $A$, and the number $L_{k}(A)$, finite or infinite; is the outer $k$-dimensional measure of the set $A$. If for each arbitrary set $W$ of extent $k$

$$
L_{k}(W)=L_{k}(A W)+L_{k}(W-A W),
$$

the set $A$ is measurable. This definition of measurability, which is based on that of Carathéodory for sets of linear extent, coincides with that of Lebesgue for $n$-dimensional sets. But not all such sets are measurable in the sense of Lebesgue. Likewise not all sets of extent $k$ are measurable in the sense of Carathéodory. An obvious example is a linear set in the plane which is nonmeasurable in the sense of Lebesgue.

The theory developed by Carathéodory for linear outer measure, and for measurability when the set $A$ is measurable, is easily shown to hold for the measure function $L_{k}(A)$. For convenient reference we recall such results of this theory as we shall have occasion to use.

CI. If the sets $A$ and $B$ are of extent $k$, and if $A$ contains $B$, then

$$
L_{k}(B) \leqq L_{k}(A) \text {. }
$$

CII. If $A$ is the set each point of which is on one of the sets $A_{1}, A_{\mathbf{2}}, \cdots$, then

$$
L_{k}(A) \leqq L_{k}\left(A_{1}\right)+L_{k}\left(A_{2}\right)+\cdots \text {. }
$$

† A space which by a proper choice of coordinate axes can be represented by $x_{1}=x_{2}=\cdots=$ $x_{n-k}=0$. A domain $U$ is convex if every $S_{2} U$ is convex. 
CIII. If $A_{1}, A_{2}, \cdots$ is a sequence of sets such that $A_{n}$ contains $A_{n-1}$, and $A$ is the limit set, then

$$
\lim _{n \rightarrow \infty} L_{k}\left(A_{n}\right)=L_{k}(A)
$$

CIV. If $A$ and $B$ are such that every point of $A$ is a distance not less than $\delta>0$ from any point of $B$, then

$$
L_{k}(A)+L_{k}(B)=L_{k}(A+B) .
$$

2. Some general lemmas. In this section we prove three lemmas.

LEMMA I. If $A$ is such that $L_{k}(A)$ is finite and different from zero, then $L_{k-1}(A)$ is infinite and $L_{k+1}(A)=0$.

That $L_{k+1}(A)=0$ follows readily from the fact that, for any set of domains $u_{i}$ with $d_{i}<\rho, \sum l_{k+1}^{i}<\rho \sum l_{k}^{i}$. We then have $L_{k-1}(A)$ infinite. For a supposition that $L_{k-1}(A)$ is finite makes $L_{k}(A)=0$.

LEMma II. $\dagger$ Let $V=V_{1}, V_{2}, \cdots$ be an infinite sequence of open convex domains in $S_{n}$, and $A$ any set of points. Then

$$
L_{k}(A V)+L_{k}(A-A V)=L_{k}(A) .
$$

First let $V$ consist of a single domain, and let $U_{1}, U_{2}, \cdots$ be a sequence of closed domains interior to $V$ and such that $U_{n}$ contains $U_{n-1}$ and $\lim U_{n}=V$. Let $A_{n}=A U_{n}$. Then $\lim A_{n}=A V$. The sets $A_{n}$ and $A-A V$ are on closed mutually exclusive domains. Hence these two sets satisfy the conditions of CIV, and it follows that

$$
L_{k}\left(A_{n}\right)+L_{k}(A-A V)=L_{k}\left(A_{n}+A-A V\right) \leqq L_{k}(A) .
$$

And since by CIII $\lim L_{k}\left(A_{n}\right)=L_{k}(A V)$ we have

$$
L_{k}(A V)+L_{k}(A-A V) \leqq L_{k}(A) .
$$

But by CII

$$
L_{k}(A V)+L_{k}(A-A V) \geqq L_{k}(A) .
$$

These two inequalities give the Lemma for $V$ a single region. The extension to the case where $V$ consists of a finite number of regions is obvious. When $V=v_{1}, v_{2}, \cdots$, set $V_{n}=v_{1}, v_{2}, \cdots, v_{n}$. Then

$$
L_{k}\left(A V_{n}\right)+L_{k}\left(A-A V_{n}\right)=L_{k}(A) \text {. }
$$

† It has been remarked by Mr. J. F. Randolph that Lemma II follows from the definition of Carathéodory for $k$-dimensional measurability, provided the open set $V$ in $S_{n}$ is considered to be $k$-dimensional measurable in the sense of Caratheodory, with infinite measure if $k<n$. In this connection we note that if $k<n$ every open set $V$ in $S_{n}$ does not satisfy the criterion of measurability which is obtained for sets of finite extent in Theorem XII of the present paper. 
The set $A-A V_{n}$ tends to $A-A V$, and the set $A V_{n}$ tends to $A V$. And since $A V_{n}$ contains $A V_{n-1}$ it follows from CIII that

Hence

$$
\lim L_{k}\left(A V_{n}\right)=L_{k}(A V) \text {. }
$$

$$
L_{k}(A V)+\lim L_{k}\left(A-A V_{n}\right)=L_{k}(A) .
$$

It follows from CI that

$$
L_{k}(A-A V) \leqq \lim L_{k}\left(A-A V_{n}\right) .
$$

Suppose the equality sign does not hold. Then from (2) we get

$$
L_{k}(A V)+L_{k}(A-A V)<L_{k}(A),
$$

which, by CII, is not true. Hence

$$
L_{k}(A V)+L_{k}(A-A V)=L_{k}(A),
$$

and the Lemma is proved.

LEMMA III. Let $V(\rho)$ denote any finite or countably infinite set of open convex domains $V_{1}, V_{2}, \cdots$ with greatest diameter $d_{i}<\rho$. Then to any set $A$ of extent $k$ and any positive number $\epsilon$ there corresponds a number $\rho_{1}>0$ such that for any set $V(\rho)$ with $\rho<\rho_{1}$ the inequality

$$
L_{k}[A V(\rho)]<\sum l_{k}^{i}+\epsilon
$$

is satisfied.

This Lemma has been proved for linearly measurable plane sets by Besicovitch. $\dagger$ His inequality (2) follows from the measurability of the set. But the corresponding inequality for any set follows from Lemma II above. The remainder of the argument is similar to that of Besicovitch with $\sum l_{k}^{i}$ replacing $\sum d_{i}$ for the various regions involved.

3. Density. Let $A$ be a set of extent $k, a$ any point of $A$, and $H(a, r)$ an $n$-dimensional hypersphere with center $a$ and radius $r$. Let $h_{k}^{r}$ be the $k$ dimensional measure of the maximal $k$-dimensional flat space that can be inscribed in $H(a, r)$. Let

$$
D(a, r)=\frac{L_{k}[A H(a, r)]}{h_{k}^{r}},
$$

and let $D^{*}(a)$ and $D_{*}(a)$ be the upper and lower limits respectively of $D(a, r)$ as $r$ tends to zero. These numbers are respectively the upper and lower densities of $A$ at $a$.

† Loc. cit., p. 427. 
It has been shown by Besicovitch $\dagger$ that the linear measure of a plane set depends on the type of region $u_{i}$ used in estimating this measure. A similar state of affairs is to be expected for sets with extent greater than unity. In estimating outer measure we shall take into consideration only types of regions $u_{i}$ which are such that

$$
l_{k}^{i}=\phi_{k} d^{k},
$$

where $\phi_{k}$ is a constant depending on $k$ and on the particular type of region, and $d$ is the greatest diameter of the region. For a hypersphere $H(a, r)$ let

$$
h_{k}^{r}=\psi_{k}(2 r)^{k} \text {. }
$$

We then have

THEOREM I. If $A$ is any set of extent $k$, then for almost all points of $A$,

$$
\frac{1}{2^{k}} \frac{\phi_{k}}{\psi_{k}} \leqq D^{*}(a) \leqq 1 .
$$

Let $E$ be the part of $A$ for each point of which $D^{*}(a)>1$. Let $E_{\lambda}$ be the part of $E$ about each point $e$ of which there exists a sequence of hyperspheres $H\left(e, r_{i}\right)$ with

$$
\frac{L_{k}\left[A H\left(e, r_{i}\right)\right]}{h_{k}^{r_{i}}}>1+\lambda .
$$

By Lemma III there exists $\rho>0$ such that for any set of hyperspheres $H_{i}$ with $r_{i}<\rho$ we have

$$
\sum L_{k}\left(E_{\lambda} H_{i}\right)<\sum h_{k}^{r_{i}}+\epsilon .
$$

From the set of hyperspheres defined in (1) let those with $r_{i}>\rho$ be discarded. It is then possible to use Vitali's argument to show the existence of a countable non-overlapping sequence of the remaining hyperspheres of $H_{i}$ which contain almost all of $E_{\lambda}$. From (1) for this sequence we have

$$
\sum h_{k} r_{i}(1+\lambda)<L_{k}\left(E_{\lambda}\right) \text {. }
$$

But from (2) we get

$$
\sum h_{k}^{r_{i}}>L_{k}\left(E_{\lambda}\right)-\epsilon .
$$

But for $\lambda$ sufficiently small $L_{k}\left(E_{\lambda}\right)>0$, and $\epsilon$ can be taken arbitrarily small independently of $\lambda$. This makes (3) and (4) contradictory, which proves that $D^{*}(a) \leqq 1$ for almost all $A$.

† Loc. cit., p. 459. 
To complete the proof of Theorem I we notice that the argument of Besicovitch $\dagger$ may be used to establish the existence of a part $A_{1}$ of $A$ with $L_{k}\left(A_{1}\right)$ arbitrarily near to $L_{k}(A)$ such that about each point $a_{1}$ of $A_{1}$ there exists a hypersphere $H\left(a_{1}, d\right)$ for which

$$
L_{k}\left\{A H\left(a_{1}, d\right)\right\} \geqq \frac{\phi_{k} d^{k}}{1+\eta},
$$

where $\eta$ is arbitrary and $d<\eta$.

From this we get, by dividing by $h_{k}{ }^{d}$,

$$
\frac{L_{k}\left[A H\left(a_{1}, d\right)\right]}{h_{k}{ }^{d}} \geqq \frac{\phi_{k} d^{k}}{h_{k}{ }^{d}}-\eta \geqq \frac{1}{2^{k}} \frac{\phi_{k}}{\psi_{k}}-\eta .
$$

Completing the argument along the lines followed by Besicovitch we finally arrive at

at almost all points of $A$.

$$
D^{*}(a) \geqq \frac{1}{2^{k}} \frac{\phi_{k}}{\psi_{k}}
$$

We note that if hyperspheres are used in computing the outer measure of $A$ then we get $1 / 2^{k}$ as a lower bound for the upper density at almost all points of $A$.

If, at a point $a$ of the set $A, D_{*}(a)=D^{*}(a)=1$, then $A$ is regular at $a$. Otherwise $A$ is irregular at $a$. The existence of sets of extent $k$ which are regular at almost every point is obvious. Besicovitch $\ddagger$ has shown the existence of linearly measurable plane sets which are irregular. An evident modification of his methods may be used to construct sets of extent $k=2$ in $S_{3}$ which are irregular.

THEOREM II. If $A$ is any set of extent $k$ then the part of $A$ for which $D^{*}(a)=0$ has zero measure, regardless of the type of region used in estimating $L_{k}(A)$.

For the sake of simplicity we prove this for a set $A$ of extent two in threedimensional space. With suitable notation the method may be used to obtain the same result for sets of extent greater than two.

Let $A_{\delta}$ be the part of $A$ for which

$$
\frac{L_{2}[A H(a, r)]}{h_{2}^{r}}<\epsilon, r<\delta .
$$

For $\delta$ sufficiently small $L_{2}\left(A_{\delta}\right)>0$. Put $A_{\delta}$ in $u_{1}, u_{2}, \ldots$ where $d_{i}<\delta / 2^{3 / 2}$, and $\sum l_{2}^{i}<L_{2}\left(A_{\delta}\right)+\epsilon$. About $u_{i}$ circumscribe a rectangular parallelepiped $p_{i}$ with

$\dagger$ Loc. cit., pp. 428-429.

$\ddagger$ Loc. cit., p. 431. 
longest side parallel to a greatest diameter $d_{i}$ of $u_{i}$. Then, since $u_{i}$ is convex, the maximal plane section of $p_{i}$ is not greater than $4 l_{2}^{i}$. Circumscribe $p_{i}$ by a cylinder $C_{i}$ with axis parallel to longest side of $p_{i}$. Then the measure $q_{i}$ of a cross section of this cylinder through the axis is not greater than $4 l_{2}$. It is evidently possible to cover a part of $C_{i}$ with cylinders $C_{i j}$ with length $t_{i j}$ and radius $r_{i j}$ where $t_{i j}=2 r_{i j} \leqq 2 d_{2}$, where $\sum q_{i j} \leqq 2 q_{i} \leqq 8 l_{2}$, and where $\sum_{i} \sum_{i}$ $L_{k}\left(A_{\delta} C_{i j}\right) \geqq L_{k}\left(A_{\delta}\right) / 2$. Fix any point of $A_{\delta}$ in $C_{i j}$, and with this point as center construct a sphere $H\left(a_{\delta}, 2 t_{i j}\right)$ with radius $2 t_{i j} . H\left(a_{\delta}, 2 t_{i j}\right)$ then contains $C_{i j}$ and $h_{2}{ }^{2 t_{i j}}=4 \pi\left(2 t_{i j}\right)^{2}=16 \pi q_{i j}$. From these and (1), since the greatest linear dimension of $C_{i j}$ is less than $2^{3 / 2} d_{i}<\delta$, we get

which gives

$$
\frac{L_{2}\left[A_{\delta} C_{i j}\right]}{h_{2}{ }^{2 t_{i j}}}<\epsilon,
$$

$$
\begin{gathered}
\sum_{i} \sum_{i} L_{2}\left(A_{\delta} C_{i j}\right) \leqq \epsilon \sum_{i} \sum_{i} h_{2}^{2 t_{i j}} \leqq \epsilon 16 \pi \sum_{i} \sum_{j} q_{i j} \leqq \epsilon 128 \pi \sum_{i} l_{2}^{\prime}, \\
\frac{\lambda}{2}<\epsilon 128 \pi\left[L_{k}\left(A_{\delta}\right)+\epsilon\right]<\frac{\lambda+\epsilon}{4} .
\end{gathered}
$$

But $\lambda$ can be fixed greater than zero, and $\epsilon$ can be taken arbitrarily small independent of $\lambda$. We are thus led to a contradiction, which proves the theorem.

4. Separated sets. A point set $A$ is separated from a point set $B$, if a part of $A$ can be put in a set of open convex regions $\alpha$ in such a way that

$$
L_{k}(\alpha B)<\epsilon \text {, and } L_{k}(A-\alpha A)<\epsilon .
$$

TheOREM III. If $A$ is separated from $B$, then $B$ is separated from $A$.

It follows from CIII that if $\alpha=\alpha_{1}, \alpha_{2}, \cdots$ satisfies (1), and if $\alpha^{\prime}=\alpha_{1}$, $\alpha_{2}, \cdots, \alpha_{n}$, then for $n$ sufficiently large

$$
L_{k}\left(\alpha^{\prime} A\right)>L_{k}(A)-2 \epsilon .
$$

Let $V_{i}=v_{i 1}, v_{i 2}, \cdots, v_{i n}$ where $v_{i j}$ is a closed region interior to $\alpha_{j}$, such that $v_{i j}$ contains $v_{(i-1)}$, and $\lim v_{i j}=\alpha_{i}$. Then by CIII, for $i$ sufficiently large, we have

$$
L_{k}\left(A V_{i}\right)>L_{k}\left(\alpha^{\prime} A\right)-\epsilon>L_{k}(A)-3 \epsilon .
$$

Put the part of $B$ exterior to $\alpha$ in $\beta$ so that any point of $\beta$ is distant from $V_{\boldsymbol{i}}$ by not less than $\delta>0$. Then

$$
L_{k}(B-\beta B) \leqq L_{k}(\alpha B)<\epsilon,
$$


and, by CIV,

$$
L_{k}\left(V_{i} A\right)+L_{k}(\beta A)=L_{k}\left(V_{i} A+\beta A\right) \leqq L_{k}(A),
$$

which, with (1), gives

$$
L_{k}(\beta A)<3 \epsilon .
$$

THEOREM IV. If $A$ and $B$ are separated sets, both of extent $k$, then

$$
L_{k}(A)+L_{k}(B)=L_{k}(A+B) .
$$

Put $A$ in a set of open convex regions $\alpha$ so that

$$
L_{k}(\alpha B)<\epsilon, L_{k}(A-\alpha A)<\epsilon .
$$

Set $E=A+B$. Then, by Lemma II,

$$
L_{k}(\alpha E)+L_{k}(E-\alpha E)=L_{k}(E),
$$

which, from CI, gives

$$
L_{k}(\alpha A)+L_{k}(B-\alpha B) \leqq L_{k}(E) .
$$

But from Lemma II we get

$$
L_{k}(\alpha A)+L_{k}(A-\alpha A)=L_{k}(A),
$$

and

$$
L_{k}(\alpha B)+L_{k}(B-\alpha B)=L_{k}(B) .
$$

It then follows from (1), (3), (4), (5), and the fact that $\epsilon$ is arbitrary, that

$$
L_{k}(A)+L_{k}(B) \leqq L_{k}(A+B) .
$$

But by CII

$$
L_{k}(A)+L_{k}(B) \geqq L_{k}(A+B) .
$$

These two inequalities give the theorem.

Let $A_{B}{ }^{0}$ be the part of $A$ for which

$$
\varlimsup_{r \rightarrow 0} \frac{L_{k}[B H(a, r)]}{L_{k}[A H(a, r)]}=0,
$$

and $A_{B}{ }^{+}$the part of $A$ for which

$$
\varlimsup_{r \rightarrow 0} \frac{L_{k}[B H(a, r)]}{L_{k}[A H(a, r)]}>0 .
$$

Define $B_{A}{ }^{0}$ and $B_{A}{ }^{+}$by interchanging the roles of $A$ and $B$. 
The ratios which are used in determining the sets $A_{B}{ }^{0}$ and $A_{B}{ }^{+}$are defined for almost all $A$. For otherwise there would exist a part of $A$ with outer measure $>0$ for each point $a$ of which $D^{*}(a)=0$. But this contradicts Theorem II. Likewise the ratios used in determining the sets $B_{A}{ }^{0}$ and $B_{A}{ }^{+}$are defined for almost all $B$.

THEOREM V. The set $A_{B}{ }^{0}$ is separated from $B$, and the set $B_{A}{ }^{0}$ is separated from $A$.

For a given $\epsilon>0$ there corresponds to each point of $A_{B}{ }^{0}$ a number $\rho>0$ such that

$$
\frac{L_{k}[B H(a, r)]}{L_{k}[A H(a, r)]}<\epsilon, r<\rho .
$$

If $E$ is the part of $A_{B}{ }^{0}$ for which (1) holds, then for $\rho$ sufficiently small it follows from CIII that

$$
L_{k}(E)>L_{k}\left(A_{B}^{0}\right)-\epsilon .
$$

About each point of $E$ there then exists a sequence of hyperspheres $H\left(e, r_{i}\right)$ with $r_{i}$ tending to zero for which (1) holds. Vitali's argument may now be used to show the existence of a non-overlapping set of these hyperspheres $H=H_{1}, H_{2}, \cdots$ which contain almost all of $E$. By (2) and Lemma II we get

$$
L_{k}\left(A_{B}{ }^{0}-H A_{B}{ }^{0}\right)<\epsilon,
$$

and from (1)

$$
\begin{aligned}
\sum L_{k}\left(B H_{i}\right) & <\epsilon \sum L_{k}\left(A H_{i}\right), \\
L_{k}(B H) & <\epsilon L_{k}(A),
\end{aligned}
$$

where $\epsilon$ is arbitrary. Thus $A_{B}{ }^{0}$ is separated from $B$. In a similar manner it may be shown that $B_{A}{ }^{0}$ is separated from $A$.

THEOREM VI. There is no part of $A_{B}{ }^{+}$with outer measure greater than zero which is separated from $B$, and no part of $B_{A^{+}}{ }^{+}$with outer measure greater than zero which is separated from $A$.

Suppose there is such a part of $A_{B}{ }^{+}$. CIII may then be used to show the existence of a positive number $d$ and a part $E$ of $A_{B}{ }^{+}$such that at each point $e$ of $E$ we have

$$
\frac{L_{k}\left[B H\left(e, r_{i}\right)\right]}{L_{k}\left[A H\left(e, r_{i}\right)\right]}>d
$$

for a properly chosen sequence of values of $r_{i}$ tending to zero. By supposition, $E$ is separated from $B$. It is, therefore, possible to put a part of $E$ in a set of 
open convex regions $\alpha$ in such a way that

$$
L_{k}(\alpha B)<\epsilon \text {, and } L_{k}(E-\alpha E)<\epsilon .
$$

About each point of $E$ on $\alpha$ may be put a sequence of hyperspheres $H\left(e, r_{i}\right)$ satisfying (1) and such that all the hyperspheres are on $\alpha$. Vitali's argument may now be used to show the existence of a countable non-overlapping set of these hyperspheres containing almost all the part of $E$ on $\alpha$. For this set $H_{n}$ of hyperspheres we get from (1)

$$
\sum L_{k}\left(B H_{n}\right)>d \sum L_{k}\left(A H_{n}\right)>d L_{k}(E)-\epsilon .
$$

But this contradicts (2). We conclude, therefore, that there is no part of $A_{B}{ }^{+}$with outer measure $>0$ which is separated from $B$. In a similar manner it can be shown that there is no part of $B_{A}{ }^{+}$with outer measure $>0$ which is separated from $A$.

It has now been shown that $A_{B}{ }^{0}$ is separated from $B$ and no part of $A_{B}{ }^{+}$is separated from $B$, with similar remarks applying to $B_{A^{0}}$ and $B_{A}{ }^{+}$. From these facts it is easy, by methods used above, to obtain

THEOREM VII. $A_{B}{ }^{0}$ is separated from $A_{B}{ }^{+}$and $B_{A}{ }^{0}$ is separated from $B_{A^{+}}$.

We next prove

Theorem VIII. $L_{k}\left(A_{B^{+}}\right)=L_{k}\left(B_{A^{+}}\right)=L_{k}\left(A_{B^{+}}+B_{A^{+}}\right)$.

Suppose $L_{k}\left(A_{B}^{+}\right)=L_{k}\left(B_{A^{+}}+\right)+c, c>0$. Making use of Lemma III, a number $\rho>0$ may be so fixed that for any set of open convex regions $V=v_{1}$, $v_{2}, \cdots$ with $d_{i}<\rho$ we have

$$
L_{k}\left(V A_{B}^{+}\right)<\sum l_{k}^{i}+\frac{c}{4} .
$$

Now let $V$ with $d_{i}<\rho$ enclose $B_{A^{+}}$in such a way that

This, with (1), gives

$$
\sum l_{k}^{i}<L_{k}\left(B_{A}^{+}\right)+\frac{c}{4} \text {. }
$$

$$
L_{k}\left(V A_{B}{ }^{+}\right)<L_{k}\left(B_{A}{ }^{+}\right)+\frac{c}{2},
$$

which shows that there is a part $E$ of $A_{B}{ }^{+}$exterior to $V$ with $L_{k}(E)>c / 2$. But $V$ contains $B_{A^{+}}$. Hence, since $E$ is exterior to $V$, it may be shown by methods used above that $E$, a part of $A_{B}{ }^{+}$with outer measure $>0$, is separated from $B$. But this contradicts Theorem VI. We conclude, therefore, that $L_{k}\left(A_{B}^{+}\right) \leqq L_{k}\left(B_{A^{+}}\right)$. Precisely the same argument shows that $L_{k}\left(B_{A^{+}}\right)$ $\leqq L_{k}\left(A_{B^{+}}\right)$. We thus have $L_{k}\left(A_{B}^{+}\right)=L_{k}\left(B_{A^{+}}\right)$, which is the first part of the theorem. 
Suppose $L_{k}\left(A_{B^{+}}+B_{A^{+}}\right)=L_{k}\left(A_{B^{+}}{ }^{+}\right)+c, c>0$. Lemma III may be used to enclose $A_{B}{ }^{+}$in a set of open convex regions $V=v_{1}, v_{2}, \cdots$ in such a way that

$$
\sum l_{k}^{i}<L_{k}\left(A_{B^{+}}^{+}\right)+\frac{c}{4}
$$

and

$$
L_{k}\left[\left(A_{B^{+}}+B_{A^{+}}\right) V\right]<\sum l_{k}^{i}+\frac{c}{4},
$$

which shows that there is a part $E$ of $A_{B}{ }^{+}+B_{A}{ }^{+}$exterior to $V$ with measure $>c / 2$. Since $V$ contains $A_{B}{ }^{+}$it follows that $E$ belongs to $B_{A^{+}}$. Reasoning as above, we arrive at the conclusion that $E$ is separated from $A_{B^{+}}$. But this again contradicts Theorem VI. Thus we conclude that $L_{k}\left(A_{B^{+}}+B_{A^{+}}\right) \leqq L_{k}\left(A_{B}{ }^{+}\right)$. Hence, since always $L_{k}\left(A_{B^{+}}+B_{A^{+}}{ }^{+} \geqq L_{k}\left(A_{B^{+}}\right)=L_{k}\left(B_{A^{+}}\right)\right.$, we have

$$
L_{k}\left(A_{B}^{+}+B_{A}^{+}\right)=L_{k}\left(A_{B}^{+}\right)=L_{k}\left(B_{A^{+}}^{+}\right) .
$$

From Theorems IV, V, and VII, we get

THEOREM IX.

$$
\begin{aligned}
L_{k}(A) & =L_{k}\left(A_{B}^{0}\right)+L_{k}\left(A_{B}^{+}\right), \\
L_{k}(B) & =L_{k}\left(B_{A}^{0}\right)+L_{k}\left(B_{A}^{+}\right), \\
L_{k}(A+B) & =L_{k}\left(A_{B}^{0}\right)+L_{k}\left(B_{A}^{0}\right)+L_{k}\left(A_{B}^{+}+B_{A}^{+}\right) .
\end{aligned}
$$

Theorems VIII and IX may now be combined to give

THEOREM X.

$$
L_{k}(A)+L_{k}(B)=L_{k}(A+B)+L_{k}\left(A_{B}{ }^{+}+B_{A}{ }^{+}\right) .
$$

5. Relations between sets in general and measurable sets. Let $A$ be any set of finite extent $k$ in $S_{n}, B$ the complement of $A$. Let $C$ be the part of $B$ for which

$$
\varlimsup_{r \rightarrow 0} \frac{L_{k}[A H(c, r)]}{h_{k}{ }^{r}}>0 .
$$

THEOREM XI. The set $C$ is of extent not greater than $k$.

Suppose there is some integer $j \geqq 1$ for which

$$
L_{k+j}(C)>0 .
$$

On account of (1) there exist two positive numbers $\delta$ and $d$, and a part $C_{1}$ of $C$ with $L_{k+i}\left(C_{1}\right)>0$ for which 


$$
\frac{L_{k}\left[A H\left(c_{1}, r\right)\right]}{h_{k}^{r}}>d
$$

for a proper choice of $r<\delta$. Since $L_{k+j}\left(C_{1}\right)>0$, it follows from Lemma I that there exists a part $C_{2}$ of $C_{1}$ with $L_{k}\left(C_{2}\right)>G, G$ an arbitrary positive number. Choose a sequence $\delta_{1}>\delta_{2}>\cdots$ tending to zero, and let $C_{2}{ }^{i}$ be the part of $C_{2}$ for which (2) holds for some $r>\delta_{i}$. Then $C_{2}{ }^{i}$ tends to $C_{2}$. Thus there exists $\delta^{\prime}>0$ and a part $C_{3}$ of $C_{2}$ with $L_{k}\left(C_{3}\right)>G$ for which we have

$$
\frac{L_{k}\left[A H\left(c_{3}, r\right)\right]}{h_{k}{ }^{r}}>d
$$

for some $r>\delta^{\prime}$. Now put $C_{3}$ in a set of open convex regions $u_{1}, u_{2}, \cdots$ with $d_{i}<\delta^{\prime}$, and such that

$$
\sum l_{k}^{i}>L_{k}\left(C_{3}\right)-\epsilon>G \text {. }
$$

In each $u_{i}$ choose a point $c_{3}$ of $C_{3}$ and about this point put a hypersphere $H\left(c_{3}, r\right)$ with $r_{i}>\delta^{\prime}$ and satisfying (3). Then $h_{k}^{i}>l_{k}^{i}$, and consequently from (3) we get

$$
\frac{L_{k}\left[A H\left(c_{3}, r_{i}\right)\right]}{l_{k^{r_{i}}}} \geqq \frac{L_{k}\left[A H\left(c_{3}, r_{i}\right)\right]}{h_{k}^{r_{i}}}>d .
$$

This gives

$$
\sum L_{k}\left[A H\left(c_{3}, r_{i}\right)\right]>d \sum l_{k}^{r_{i}}>d G .
$$

But since $L_{k}(A)$ is finite, and since $G$ can be chosen arbitrarily large, this gives a contradiction. Hence our assertion is proved.

THEOREM XII. If $A$ is any set of finite extent $k$, then a necessary and suffcient condition that $A$ be measurable is that $L_{k}(C)=0$.

Let $W$ be any set of extent $k$. We show that if $L_{k}(C)=0$, then

$$
L_{k}(W)=L_{k}(A W)+L_{k}(W-A W) .
$$

Set $W-A W=E$. Since $E-E C$ belongs neither to $A$ nor to $C$, we have for any point $e$ of $E-E C$

$$
\varlimsup_{r \rightarrow 0} \frac{L_{k}[A H(e, r)]}{h_{k}{ }^{r}}=0 .
$$

Hence, since $L_{k}(C)=0$, for almost all $E$ we have

$$
\varlimsup_{r \rightarrow 0} \frac{L_{k}[A H(e, r)]}{L_{k}[E H(e, r)]} / \frac{h_{k}^{r}}{L_{k}[E H(e, r)]}=0 .
$$


But for almost all $E$

$$
\varlimsup_{r \rightarrow 0} \frac{h_{k}{ }^{r}}{L_{k}[E H(e, r)]} \geqq 1,
$$

and this, with (2), gives, for almost all $E$,

$$
\varlimsup_{r \rightarrow 0} \frac{L_{k}[A H(e, r)]}{L_{k}[E H(e, r)]}=0 .
$$

Hence almost all $E-E C$ belongs to $W_{A^{0}}$. And since $L_{k}(E C)=L_{k}(C)=0$, it follows that almost all $E$ belongs to $W_{A}{ }^{0}$. Hence $E=W-A W$ is separated from $A$ and consequently from $W A$. The truth of (1) now follows from Theorem IV. Then, according to our definition of measurability, $A$ is measurable. Thus the condition is sufficient.

At every point of $C$

$$
\varlimsup_{r \rightarrow 0} \frac{L_{k}[A H(c, r)]}{h_{k}^{r}}>0 .
$$

Hence for almost all $C$

$$
\varlimsup_{r \rightarrow 0} \frac{L_{k}[A H(c, r)]}{L_{k}[C H(c, r)]} / \frac{h_{k}{ }^{r}}{L_{k}[C H(c, r)]}>0 .
$$

But for almost all $C$

$$
\varliminf_{r \rightarrow 0} \frac{h_{k}^{r}}{L_{k}[C H(c, r)]} \geqq 1 .
$$

Consequently, for almost all $C$,

$$
\varlimsup_{r \rightarrow 0} \frac{L_{k}[A H(c, r)]}{L_{k}[C H(c, r)]}>0 .
$$

We conclude, therefore, that almost all $C$ belongs to $C_{A^{+}}$. Hence if $L_{k}(C)>0$ it follows that $L_{k}\left(C_{A^{+}}\right)>0$. Now let $W=A_{C^{+}}+C_{A^{+}}$. Then by Theorem VIII,

$$
L_{k}(W)=L_{k}\left(A_{C^{+}}\right)=L_{k}\left(C_{A^{+}}\right) \text {. }
$$

But

$$
L_{k}(A W)+L_{k}(W-A W)=L_{k}\left(A_{C^{+}}^{+}\right)+L_{k}\left(C_{A}^{+}\right)=2 L_{k}(W) .
$$

Hence $A$ is not measurable. This shows that the condition is necessary.

Theorem XIII. Let $A$ be any set of finite extent $k$. Then the set

$$
A^{\prime}=A+C
$$

contains $A$, is measurable with 


$$
L_{k}\left(A^{\prime}\right)=L_{k}(A),
$$

and is well-defined in terms of $A$.

A point $b$ of the set $B$ complementary to the set $A$ does or does not belong to $C$ according as the upper limit of

$$
\frac{L_{k}[A H(b, r)]}{h_{k}^{r}}
$$

is greater than zero, or is equal to zero. Hence $C$, and consequently $A^{\prime}$, is effectively defined in terms of $\mathrm{A}$.

To show that $A^{\prime}$ is measurable, let $c^{\prime}$ be a point of $C^{\prime}$. Then $c^{\prime}$ belongs neither to $A$ nor to $C$, and

$$
\varlimsup_{r \rightarrow 0} \frac{L_{k}\left[A^{\prime} H\left(c^{\prime}, r\right)\right]}{h_{k}^{r}}>0 .
$$

But this, with Theorems IV, VII, and VIII, gives

$$
\begin{gathered}
\lim _{r \rightarrow 0}\left\{\frac{L_{k}\left[A_{\left.C^{0} H\left(c^{\prime}, r\right)\right]}\right.}{h_{k}^{r}}+\frac{L_{k}\left[\left(A_{C^{+}}+C_{A^{+}}\right) H\left(c^{\prime}, r\right)\right]}{h_{k}^{r}}\right\}>0, \\
\varlimsup_{r \rightarrow 0} \frac{L_{k}\left[\left(A_{C^{0}}+A_{C^{+}}\right) H\left(c^{\prime}, r\right)\right]}{h_{k}^{r}}>0,
\end{gathered}
$$

which makes $c^{\prime}$ a point of $C$. Hence $C^{\prime}$ is empty and $L_{k}\left(C^{\prime}\right)=0$. Then by Theorem XII $A$ is measurable.

THEOREM XIV. If the set $A$ of extent $k$ is regular then $A^{\prime}$ is regular, and if this set is irregular then $A^{\prime}$ is irregular.

Let $A$ be regular, and suppose there is a part of $A^{\prime}$, other than null parts, at which $A^{\prime}$ is irregular. Obviously $A^{\prime}$ is regular at each point of $A$. In the proof of Theorem XII it was shown that almost all $C$ belongs to $C_{A^{+}}$. Hence there is a part $E$ of $C_{A}{ }^{+}$for which

$$
\frac{L_{k}\left[A^{\prime} H(e, r)\right]}{h_{k}^{r}}<1-\eta
$$

for an infinite set of arbitrarily small $r, \eta>0$, and $L_{k}(E)>0$. Then, since $E$ belongs to $C_{A^{+}}$, almost all points of $E$ are points of $E_{A^{+}}$. This and Theorem VIII then give

$$
L_{k}\left(A_{E^{+}}\right)=L_{k}\left(E_{\Lambda}^{+}\right)=L_{k}(E)>0 .
$$

The set $A^{\prime}$ is regular at points of $A$ and consequently at points of $A_{E^{+}}$. Let 
$F$ be the part of $A_{E}^{+}$which is such that

$$
\frac{L_{k}\left[A^{\prime} H(f, r)\right]}{h_{k}{ }^{r}}>1-\frac{\eta}{2}
$$

for $r<\delta, L_{k}(F)>0$. The set $F$ belongs to $A_{E^{+}}$, and consequently almost all $F$ belongs to $F_{E^{+}}$. Hence, as above,

$$
L_{k}\left(E_{F}^{+}\right)=L_{k}\left(F_{E^{+}}^{+}\right)=L_{k}(F)>0 .
$$

For each point of $E_{F^{+}}$,(1) holds. Hence about a fixed point $x$ of this set there exists $H(x, r)$ with $r<\delta$ such that

$$
\frac{L_{k}\left[A^{\prime} H(x, r)\right]}{h_{k}{ }^{r}}<1-\eta .
$$

Every point of $E_{F}{ }^{+}$is a limit point of points of $F_{E^{+}}$. Let $x_{1}, x_{2}, \cdots$ be a sequence of points of $F_{E}+$ tending to $x$. On account of (2) for an arbitrary $\epsilon$ there exists $H\left(x_{i}, r-\epsilon\right)$ about each $x_{i}$ for which

$$
\frac{L_{k}\left[A^{\prime} H\left(x_{i}, r-\epsilon\right)\right]}{h_{k}^{r-\epsilon}}>1-\frac{\eta}{2} \text {. }
$$

For $\epsilon$ fixed and $i$ sufficiently large $H\left(x_{i}, r-\epsilon\right)$ is interior to $H(x, r)$. From this and (4) we have

$$
L_{k}\left[A^{\prime} H(x, r)\right] \geqq L_{k}\left[A^{\prime} H\left(x_{i}, r-\epsilon\right)\right]>h_{k}^{r-\epsilon}-\frac{1}{2} \eta h_{k}{ }^{r-\epsilon} .
$$

But for $\epsilon$ sufficiently small $h_{k}^{r-\epsilon}$ is arbitrarily near to $h_{k}^{r}$, which makes (5) and (3) contradictory. We conclude, therefore, that if $A$ is regular $A^{\prime}$ is regular.

The proof for the case when $A$ is irregular is along the same lines, and we merely sketch it. Except for a null set

$$
A^{\prime}=A_{C^{0}}+A_{C^{+}}+C_{A}^{+} \text {. }
$$

Since $A_{C^{0}}$ is separated from $C_{A}{ }^{+}$it readily follows that $A^{\prime}$ is irregular at each point of $A c^{0}$. Also, since for any $H\left(a_{c}^{+}, r\right)$,

$$
L_{k}\left[\left(A_{C^{+}}^{+}+C_{A^{+}}^{+}\right) H\left(a_{c}^{+}, r\right)\right]=L_{k}\left[A_{C}^{+} H\left(a_{c}^{+}, r\right)\right],
$$

it follows that $A^{\prime}$ is irregular at each point of $A_{C^{+}}$. Let $E$ be the part of $C$ at which $A^{\prime}$ is regular. Let $F$ be the part of $A_{E^{+}}$for which

$$
\frac{L_{k}\left[A^{\prime} H(f, r)\right]}{h_{k}{ }^{r}}<1-\eta
$$

for an infinite set of arbitrarily small $r$. Let $G$ be the part of $E_{F}^{+}$at which, for all $r<\delta$, 


$$
\frac{L_{k}\left[A^{\prime} H(g, r)\right]}{h_{k}^{r}}>1-\frac{\eta}{2} .
$$

Then for $F_{G^{+}}$, (1) holds, and for $G_{F^{+}}$, (2) holds. It is now possible to take a point $x$ of $F_{G^{+}}$, and a sequence of points $x_{1}, x_{2}, \cdots$ of $G_{F}+$ tending to $x$, and arrive at a contradiction, as in the case when $A$ was regular.

6. Some applications. It was shown in the introduction that corresponding to a set $A$ of linear extent there was a measurable set $\bar{A}$ which contained $A$, and for which

$$
L_{k}(\bar{A})=L_{k}(A) .
$$

It can likewise be shown that there is a measurable set $\bar{A}$ similarly related to any set $A$ of extent $k$. We are now in a position to discuss the density properties of this set $\bar{A}$.

If $A$ is regular (irregular) then $\bar{A}$ is regular (irregular).

To prove this, set $\bar{A}=A+B$ where $L_{k}(B)>0$. If $L_{k}(B)=0$ the case is trivial. The set $B$ is not separated from $A$. For then we would have

$$
L_{k}(\bar{A})=L_{k}(A)+L_{k}(B)
$$

which cannot hold, since $L_{k}(\bar{A})=L_{k}(A)$. Hence almost all $B$ belongs to $B_{A^{+}}$. Now suppose that $A$ is regular but that there is a part of $B_{A}{ }^{+}$with outer measure greater than zero at which $A$ is irregular. Let $E$ be the part of $B_{A^{+}}$ at which

$$
\frac{L_{k}[\bar{A} H(e, r)]}{h_{k}{ }^{r}}<1-\eta
$$

for a sequence of values of $r$ tending to zero, and $L_{k}(E)>0$. Since $E$ belongs to $B_{A}^{+}$, each point of $E$ is a point of $E_{A}{ }^{+}$. Let $F$ be the part of $A_{E^{+}}$which is such that

$$
\frac{L_{k}[\bar{A} H(f, r)]}{h_{k}^{r}}>1-\frac{\eta}{2}
$$

for $r<\delta$, and $L_{k}(F)>0$. Each point of $F$ is a point of $F_{A^{+}}$. About a point $x$ of $E_{F}{ }^{+}$put a hypersphere $H(x, r)$ with $r<\delta$, and such that

$$
\frac{L_{k}[\bar{A} H(x, r)]}{h_{k}{ }^{r}}<1-\eta .
$$

Let $x_{i}$ be a sequence of values of $F_{E}+$ tending to $x$. About each $x_{i}$ put a hypersphere $H\left(x_{i}, r-\epsilon\right)$. Then, on account of (1), we have

$$
\frac{L_{k}\left[\bar{A} H\left(x_{i}, r-\epsilon\right)\right]}{h_{k}^{r-\epsilon}}>1-\frac{\eta}{2} \text {. }
$$


For any $\epsilon, i$ can be taken large enough to insure that $H\left(x_{i}, r-\epsilon\right)$ is interior to $H(x, r)$. But for $\eta$ fixed, $\epsilon$ can be taken arbitrarily small, which, with (2), (3), and the fact that for $\epsilon$ small $h_{k}^{r-\epsilon}$ is near to $h_{k}^{r}$, leads to a contradiction. We conclude, therefore, that if $A$ is regular then $\bar{A}$ is regular at almost all points. Similar reasoning shows that if $A$ is irregular then $\bar{A}$ is irregular at almost all points.

If $A$ is any plane set of linear extent then the sets $\bar{A}$ and $A^{\prime}$ are linearly measurable, and are regular or irregular according as $A$ is regular or irregular. Besicovitch has shown that linearly measurable regular plane sets have a tangent at almost all points. Hence if $A$ is regular there exists a tangent at almost all points of $\bar{A}$, and of $A^{\prime}$. And since each of these sets contains $A$ it follows that $A$ has a tangent at almost all points. Likewise the other theorems which Besicovitch has proved for linearly measurable plane sets are seen to hold for general sets of linear extent.

In proving that a regular linearly measurable plane set $A$ has a tangent at almost every point, Besicovitch $\dagger$ makes use of the set $A_{1}$ which is the part of $A$ for which

$$
\left|\frac{L_{1}[A H(a, r)]}{h_{1}{ }^{r}}-1\right| \leqq \eta
$$

for $r<\delta$. He assumes that $A_{1}$ is linearly measurable. The measurability of this set can hardly be considered as obvious, and there seems to be no trivial proof for his assertion. We shall establish some general results from which the measurability of $A_{1}$ follows.

We show first that

Separated divisions of measurable sets are measurable.

Let $A$ be any measurable set of extent $k, A_{1}$ and $A_{2}$ separated divisions of $A$. Since $A_{1}$ and $A_{2}$ are separated, $C_{1}$ contains at most a null part of $A_{2}$, and $C_{2}$ contains at most a null part of $A_{1}$. Hence, except for at most a null set, $C_{1}$ and $C_{2}$ belong to $C$. But since $A$ is measurable, Theorem XII gives $L_{k}(C)=0$. But this makes $L_{k}\left(C_{1}\right)=L_{k}\left(C_{2}\right)=0$, which again by Theorem XII makes $A_{1}$ and $A_{2}$ measurable.

Let $A$ be any set of extent $k$. Let $B$ be the part of $A$ for which

$$
\frac{L_{k}[A H(a, r)]}{h_{k}^{r}}>1-\eta
$$

for $r<\delta$. Then the sets $B$ and $E=A-B$ are separated.

$\dagger$ Loc. cit., p. 438. 
Suppose $B_{E^{+}}$exists with $L_{k}\left(B_{E^{+}}\right)>0$. Then, by Theorem VIII, $L_{k}\left(E_{B^{+}}\right)$ $=L_{k}\left(B_{E^{+}}{ }^{+}\right)>0$. For each point $x$ of $E_{B}{ }^{+}$there exists some $r<\delta$ for which

$$
\frac{L_{k}[A H(x, r)]}{h_{k}^{r}} \leqq 1-\eta .
$$

Take a sequence of points $x_{1}, x_{2}, \cdots$ of $B_{E^{+}}+$tending to $x$, and about each $x_{i}$ put a hypersphere $H\left(x_{i}, r-\epsilon\right)$. Then from (1) we have

$$
\frac{L_{k}\left[A H\left(x_{i}, r-\epsilon\right)\right]}{h_{k}^{r-\epsilon}}>1-\eta .
$$

But, for every $\epsilon, i$ can be taken so large that $H\left(x_{i}, r-\epsilon\right)$ is interior to $H(x, r)$. Then, since $\epsilon$ can be taken arbitrarily small independent of $\eta,(2)$ and (3) are contradictory, which allows us to conclude that $B$ and $E=A-B$ are separated.

It can likewise be shown that the part of $A$ for which

$$
1-\eta \leqq \frac{L_{k}[A H(a, r)]}{h_{k}^{r}} \leqq 1+\eta
$$

is separated from the remainder of $A$. From this it follows that the set $A_{1}$ of Besicovitch is separated from $A-A_{1}$. Then, since $A$ is measurable, $A_{1}$ and $A-A_{1}$ are measurable. We note further that if $A$ is not measurable the sets $A_{1}$ and $A-A_{1}$ are, nevertheless, separated. This fact permits the arguments of Besicovitch in regard to tangency to be carried through for any plane set of linear extent.

ACADIA UNIVERSITY,

Wolfville, Nova Scotia 